Clinical Study

\title{
Evaluation of Functional Outcomes and OCT-Biomarkers after Intravitreal Dexamethasone Implant for Postoperative Cystoid Macular Edema in Vitrectomized Eyes
}

\author{
Sigrid Freissinger, Efstathios Vounotrypidis $(D$, Armin Wolf, Karsten U. Kortuem (D), \\ Mehdi Shajari, Filippos Sakkias, Tina Herold, Siegfried G. Priglinger, \\ and Wolfgang J. Mayer
}

Department of Ophthalmology, Ludwig-Maximilians-University, Munich, Germany

Correspondence should be addressed to Efstathios Vounotrypidis; evounotr@med.lmu.de

Received 16 December 2019; Accepted 27 March 2020; Published 28 April 2020

Academic Editor: Takayuki Baba

Copyright (c) 2020 Sigrid Freissinger et al. This is an open access article distributed under the Creative Commons Attribution License, which permits unrestricted use, distribution, and reproduction in any medium, provided the original work is properly cited.

\begin{abstract}
Purpose. To evaluate the efficacy of dexamethasone implant (DEX) for the treatment of postoperative cystoid macular edema (PCME) in vitrectomized eyes and to investigate visual and morphological OCT predictive factors. Methods. In this retrospective study, eyes with PCME after vitrectomy were treated with at least one DEX injection and were observed over 12 months. Indications for surgery were epiretinal membrane (ERM) or rhegmatogenous retinal detachment (RRD) without macular involvement. Prior treatments, if any, were noted. Best corrected visual acuity (BCVA), central foveal thickness (CFT), and OCT morphology including the presence of intraretinal cysts/fluid or subretinal fluid (IRF/SRF) and ellipsoid zone (EZ) continuity were evaluated. Correlations between OCT measures and visual outcomes were analyzed by the generalized estimating equations procedure. Results. Forty-six eyes with ERM and 15 eyes with RRD were enrolled. The ERM group was more likely to gain BCVA than RRD (odds ratio (OR), 1.168; 95\% confidence interval (CI), 1.003-1.360; $p=0.046)$. The absence of SRF (OR, 0.860; 95\% CI, $0.743-0.995 ; p=0.043)$ was predictive of worse BCVA, whereas the integrity of EZ (OR, 1.094; 95\% CI, 0.951-1.257; $p=0.209)$ or naïve status (OR, 0.946; 95\% CI, 0.871-1.137, $p=0.853$ ) was not. Eyes with a worse baseline BCVA were more likely to gain $>1$ line after 12 months (OR, 1.485; 95\% CI, 1.171-1.884; $p=0.001)$. Conclusion. The efficacy of the treatment of PCME in vitrectomized eyes seems to be affected by baseline BCVA, the absence of SRF, and the indication for surgery. Naïve status appears not to play any significant role in the prediction of BCVA. This trial is registered with DRKS00018955.
\end{abstract}

\section{Introduction}

Postoperative cystoid macular edema (PCME) is one of the major reasons for visual impairment after cataract surgery, vitrectomy, or combined phacovitrectomy and usually occurs within 4 to 12 weeks [1]. In most of the cases, it resolves without treatment, but if no resorption occurs, structural changes of the retinal layers may lead to irreversible visual deterioration [2]. The pathomechanism of PCME is considered to be multifactorial [3-5], and to date, intraoperative complications (i.e., posterior capsule rupture), epiretinal membrane, vein occlusion, topical prostaglandin therapy, diabetes, and uveitis have been identified as risk factors $[6,7]$.

Treatment of PCME consists of topical nonsteroidal anti-inflammatory drugs (NSAIDs) and periocular, systemic, or intravitreal cortisone $[6,8]$. In general, topical eye drops are applied as the initial treatment, followed by periocular and intravitreal corticosteroids [6], as they offer several advantages compared with systemic corticosteroids $[9,10]$. However, their disadvantage is their short-lasting effect [11]. The faster clearance in vitrectomized eyes also reduces the effect of intravitreal steroids in comparison with nonvitrectomized eyes [12]. 
The longer-lasting single sustained-release dexamethasone intravitreal implant (Ozurde ${ }^{\circledR}$, Allergan Inc., Irvine, CA, USA, and Allergan Pharmaceuticals, Ireland) is used to achieve steroidal shielding in PCME cases [13, 14]. It is maintained up to 180 days after administration $[15,16]$; its effect lasts up to six months after injection, even in vitrectomized eyes $[17,18]$, and its efficacy and unaffected pharmacokinetic has been demonstrated in experimental and clinical trials in nonvitrectomized and vitrectomized eyes [15, 19-21].

Until now, dexamethasone intravitreal implant (DEX) has been widely used for the treatment of diabetic macular edema, noninfectious uveitis, and secondary macular edema after retinal vein occlusion [20, 22-24]. However, during the last few years, it has been more favorably applied for the treatment of PCME, regardless of preoperative diagnosis [10, 25-27].

The efficacy of DEX in the treatment of PCME after vitrectomy for ERM has been reported in several case studies [27-29] and in two large retrospective studies [30-32], whereas only two studies have evaluated DEX after vitrectomy for RRD repair [31, 33]. However, no study has as yet evaluated any visual or morphological OCT biomarkers for their predictive value in the treatment of PCME in vitrectomized eyes.

This retrospective study was conducted to evaluate the long-term safety and efficacy of DEX for the treatment of PCME in vitrectomized eyes after surgery for ERM removal or RRD repair. Furthermore, various parameters were evaluated for their influence in visual outcome one year after the first DEX injection. OCT morphology including the presence of subretinal fluid (SRF), intraretinal fluid (IRF), ellipsoid zone (EZ) continuity, central retinal thickness (CRT), prior treatments, duration between vitrectomy and onset of PCME, duration between vitrectomy and initial DEX injection, lens status at time of vitrectomy, IOP-increase, and PCME recurrence and persistence were evaluated over a period of 12 months.

\section{Materials and Methods}

2.1. Design. A retrospective longitudinal case series was established at the Department of Ophthalmology in Ludwig Maximilians University, Munich. The study was approved by the institutional review board of the Department of Ophthalmology, Ludwig Maximilians University, Munich, and adhered to the tenets of the Declaration of Helsinki (registration trial: DRKS00018955). Based on an electronic database warehouse, all vitrectomized eyes that developed PCME after surgery including treatment with at least one DEX injection and a minimum follow-up period of 12 months were selected. The indication for surgery was restricted to epiretinal membrane (ERM) or rhegmatogenous retinal detachment (RRD) without macular involvement. All eyes were pseudophakic after surgery and had an in-the-bag intraocular lens. Postoperative cystoid macular edema was diagnosed clinically and by means of spectral-domain optical coherence tomography (OCT, Spectralis, Heidelberg Engineering, Heidelberg, Germany).
Patients with previous vitrectomy, residual ERM, postoperative retinal detachment, or further intraocular surgeries during the follow-up period or topical therapy with prostaglandins were excluded. Additionally, eyes with any underlying retinal pathology that could affect the development of a cystoid macular edema, such as previous ocular trauma, age-related macular degeneration (AMD), diabetes, retinal vascular occlusion (RVO), vitreous hemorrhage, uveitis, silicon oil filling, or proliferative vitreoretinopathy, were excluded.

\subsection{Surgical Procedure. Pars plana vitrectomy (23 gauge)} included core vitrectomy, posterior vitrectomy, and vitreous base shaving. Internal limiting membrane peeling by using dye (brilliant blue) and Eckart forceps was performed in all cases. PFCL was used only in RRD cases. Balanced salt solution (BSS) was injected as a tamponade for ERM cases, whereas a gas tamponade (C2F6-15\%) was used for RRD repair. Moreover, laser coagulation was always performed after RRD surgery and when required in the other cases. Postoperative treatment included combined antibiotics and steroid eye drops 4 times a day, reduced by 1 eye drop every week.

The injection of the DEX implant was administered under local anesthesia (topical lidocaine) and sterile conditions according to the manufacturer's recommendations by using the provided 22-gauge injecting applicator. Injection was performed in the inferotemporal or superotemporal quadrant.

The implant was injected in the treatment naïve eyes and in cases with PCME that had previously been treated with parabulbous steroid injections (40 mg triamcinolone) and/ or topical nonsteroidal anti-inflammatory eye drops (nepafenac $1 \mathrm{mg} / \mathrm{ml}$ three times daily).

2.3. Examinations. Several variables were recorded and analyzed including patient demographics, lens status at surgery, indication for surgery, time between surgery and onset of PCME, time between surgery and first DEX injection, prior treatments, and number of DEX implants within the follow-up period.

Best corrected visual acuity (BCVA), optical coherence tomography (OCT, Spectralis, Heidelberg Engineering $\mathrm{GmbH}$ ), clinical examination including fundus biomicroscopy, and intraocular pressure (IOP) measured by Goldmann applanation tonometry were assessed prior to the initial DEX injection and one year after the first DEX injection. BCVA was assessed with Snellen optotypes. Central retinal thickness (CRT) involving the central foveal area of the Early Treatment Diabetic Retinopathy Study (ETDRS) macular grid was obtained. The segmentation of the retinal thickness between Bruch's membrane and the retinal nerve fiber layer had been previously noted and, if necessary, manually adjusted. OCT-morphological changes including the presence of intraretinal cysts/fluid (IRF), subretinal fluid (SRF), and EZ continuity were documented by an experienced physician (SF) and analyzed. 
The eyes were separated into two groups with regard to the indication for surgery (ERM or RRD) or prior treatment to the first DEX injection (naïve or previously treated). Further parameters such as recurrence or persistence of PCME were evaluated. Recurrence of PCME was defined as an increase of CRT $>400$ microns and the presence of IRF and/or SRF. The persistence of PCME was defined as no decrease of CRT compared with the time of initial DEX injection and the presence of IRF and/or SRF, despite multiple DEX injections.

Study-specific adverse events of the single sustained dexamethasone implant were reported. In particular, a patient with an elevated intraocular pressure $(>25 \mathrm{mmHg})$ after injection with the necessary prescription of IOPlowering therapy was assessed as a steroid responder.

2.4. Statistical Analysis. Statistical analysis was performed by using SPSS Statistics 24 (IBM, Armonk, NY, USA). Normal distribution was tested with the Kolmogorov-Smirnov test. Parametrical and nonparametrical tests were performed for BCVA and OCT data between the baseline and final followup. Data are presented as the mean \pm standard deviation (range). A value of $p<0.05$ was considered statistically significant.

The generalized estimating equation (GEE) procedure was used to calculate differences in functional treatment response between naïve and refractory patients and included baseline BCVA (prior to the initial DEX injection) as a covariate. An improvement of BCVA of more than 0.1 $\log$ MAR to baseline was classified as visual gain, a change within $\pm 0.1 \log$ MAR as no change, and a loss of more than $0.1 \log$ MAR as visual loss.

The GEE model for outcome at 12 months was run by testing the following predictive factors at baseline: (1) BCVA; (2) indication for surgery; (3) lens status; (4) naïve status; (5) presence of IRF; (6) presence of SRF; (7) EZ continuity; (8) duration between surgery and onset of PCME; (9) duration between surgery and first DEX injection. Predictors were entered into the model and kept within it if the $p$ value was less than 0.10. The final GEE model was used to calculate the odds ratios (ORs) and their 95\% confidence intervals (CIs), with a change of $0.1 \log$ MAR in baseline BCVA being considered as a standard unit of change. Values are presented as the mean \pm standard deviation (95\% CI).

\section{Results}

A total of 61 eyes (30 right/31 left) of 61 patients (34 women, 27 men) were included in the study. The mean age of the patients was $64.8 \pm 10.2$ years (35-90). Indication for surgery was ERM in 46 eyes (24 phakic, 22 pseudophakic) and RRD in 15 eyes ( 6 phakic, 9 pseudophakic).

Table 1 presents the demographic data, indication for surgery, lens status, previously treated eyes, mean number of injections, mean duration between surgery and onset of PCME, and mean duration between the diagnosis of PCME and first DEX injection.
The mean number of DEX injections was $1.57 \pm 0.7$. The number of eyes that required a second or a third injection progressively declined during the follow-up period, regardless of the indication for surgery or naïve status. The percentage of eyes that received 1, 2, or 3 injections, with regard to the indication for surgery, is demonstrated in Figure 1 and with regard to naive status is shown in Figure 2.

In total, 43 eyes $(70.5 \%)$ were treated prior to the first DEX injection, whereas 18 eyes $(29.5 \%)$ were initially treated with DEX. Thirty-three eyes (23 ERM and 10 RRD) had previously been treated with parabulbous triamcinolone injection, and 10 eyes (8 ERM and 2 RRD) with topical nonsteroidal anti-inflammatory eye drops. In ten cases previously treated, PCME was unresponsive (6 ERM and 4 RRD), and in the rest of 33 cases, PCME was recurrent. Twenty-one patients were receiving antihypertensive medication at the time of the DEX injection, but no retinal signs of hypertension were present at any time during the study.

3.1. Recurrence, Persistence, and OCT Morphology of PCME. Of the 35 eyes that received one DEX injection (23 ERM and 12 RRD), four eyes showed PCME after 12 months. Two eyes presented a recurrence (1 ERM and 1 RRD), with massive IRF, increase of CRT, and visual impairment, whereas two eyes (1 ERM and 1 RRD) had a persisting PCME without any change of BCVA and a CRT >510 microns. The eyes with persisting PCME showed a completely disrupted EZ during the last follow-up examination.

Of the 17 eyes that received two DEX injections (15 ERM and 2 RRD), four presented a PCME at the last follow-up. A recurrence was diagnosed in three cases (3 ERM). In one eye, a persisting PCME was observed with a CRT of 400 microns and no change of BCVA (ERM).

Similarly, of the nine eyes (8 ERM and 1 RRD) that received three DEX injections, three eyes showed a recurrence (2 ERM and $1 \mathrm{RRD}$ ) and one eye showed a persisting PCME with a completely disrupted EZ (ERM).

At the last follow-up examination, 49 eyes (80.3\%) showed no PCME. Of the 19 eyes that presented with subretinal fluid prior to the first DEX injection, only five (8.2\%) showed a subretinal fluid at the last follow-up examination ( $p=0.004, \mathrm{Mc}-\mathrm{Nemar}$ test). Prior to the first DEX injection, EZ was intact in 12 eyes (19.7\%), partially disrupted in 31 eyes (50.8\%), and completely disrupted in 18 eyes (29.5\%). At the last follow-up, 31 eyes (50.8\%) presented with an intact EZ, 23 eyes (37.7\%) with a partially disrupted EZ, and 7 eyes (11.5\%) with a completely disrupted EZ ( $p=0.001$, Cochran's $Q$ test $)$.

3.2. Visual Acuity and Central Retinal Thickness. In the ERM group, BCVA improved from $0.69 \pm 0.21(0.3-1.1)$ to $0.46 \pm 0.29(0.1-1.1) \log$ MAR $(p<0.0001$, Wilcoxon test $)$ and CRT decreased from $512.3 \pm 125$ (285-740) to $369.6 \pm 108.3 \mu \mathrm{m}(225-733)(p<0.0001$, Wilcoxon test). The improvement of BCVA in the RRD group from $0.58 \pm 0.17$ $(0.3-0.8)$ to $0.53 \pm 0.34(0-1.2) \log$ MAR was not statistically significant $(p=0.542$, paired $t$-test), although CRT decreased from $457.1 \pm 85.2(302-639)$ to $377.6 \pm 99.6 \mu \mathrm{m}$ 
TABLE 1: Demographic data, lens status, prior treatments, mean number of injections, mean duration between surgery and onset of PCME, and between the diagnosis of PCME and first DEX injection in all eyes, and with regard to the indication for surgery.

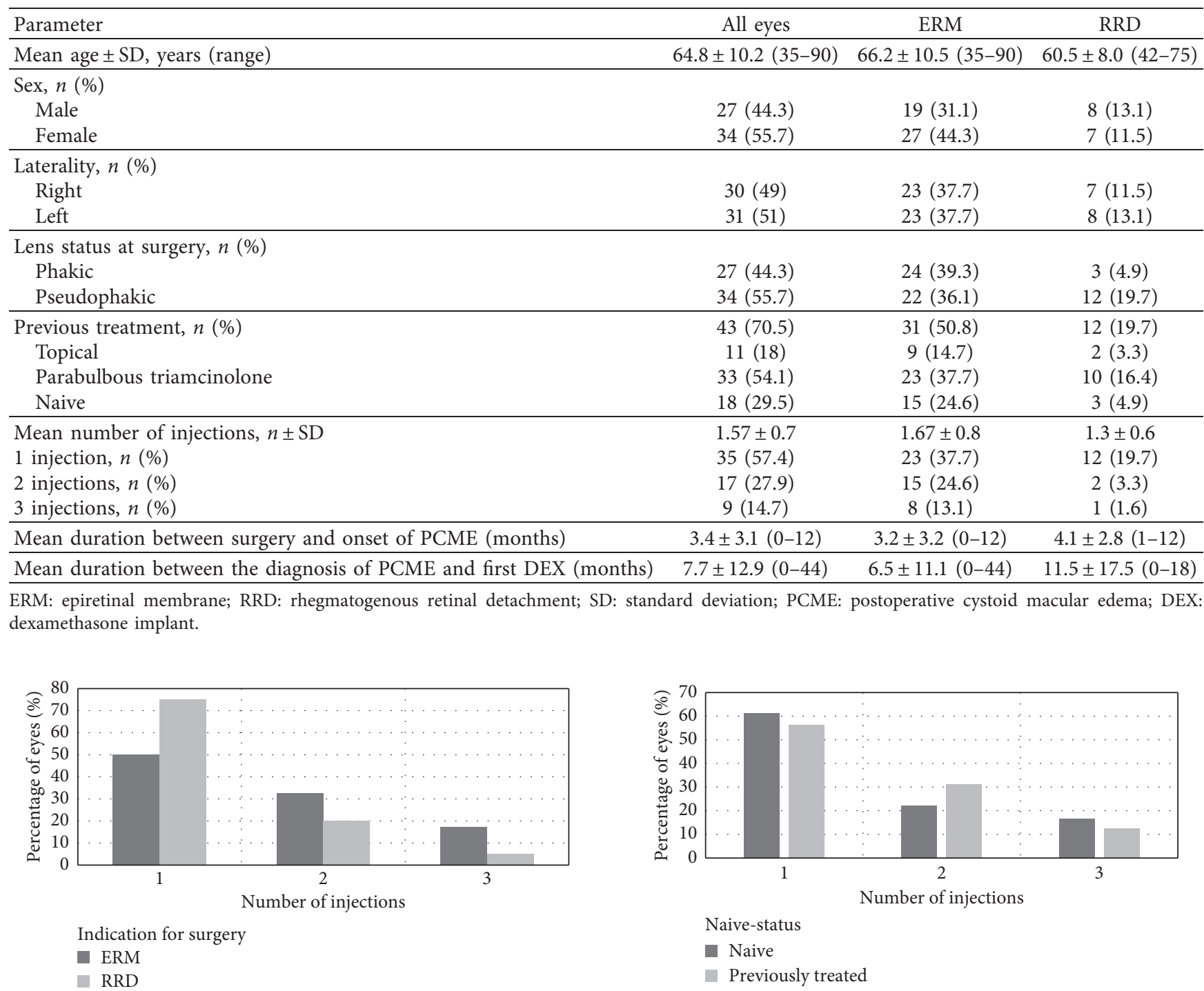

Figure 1: Percentage of eyes that received 1, 2, or 3 DEX injections with regard to indication for surgery. The percentage of eyes progressively declined with increasing number of injections for both indications for surgery. ERM: epiretinal membrane, RRD: rhegmatogenous retinal detachment, and DEX: dexamethasone implant.

(245-566) ( $p=0.016$, paired $t$-test). Table 2 demonstrates BCVA and CRT at baseline and after one year with regard to indication for surgery and prior treatments.

The percentage of eyes that showed a visual gain, loss, or no change with regard to diagnosis and prior treatment is demonstrated in Figure 3 for all eyes.

3.3. Predictive Factors. The results of the GEE with regard to the odd ratios of predictors of good functional treatment response after 12 months are presented in Table 3. Indication for surgery, absence of SRF, and baseline BCVA seem to have a significant effect on the final outcome, whereas lens
FIgure 2: Percentage of eyes that received 1, 2, or 3 DEX injections with regard to naïve status. The percentage of eyes progressively declined with increasing number of injections regardless of naïve status.

status at surgery, EZ integrity, presence of IRF, and number of DEX injections do not seem to influence the improvement of BCVA one year after the initial DEX injection. The odds gain in BCVA was increased in eyes that underwent surgery because of ERM compared with RRD (OR, 1.168; 95\% CI, $1.003-1.360 ; p=0.046)$ and in eyes with a worse baseline BCVA (OR, 1.485; 95\% CI, 1.171-1.884; $p=0.001$ ). Absence of SRF at baseline examination was a predictive factor for loss in BCVA (OR, 0.860; 95\% CI, 0.743-0.995; $p=0.043$ ). In a subanalysis, in which eyes were excluded that showed no response to DEX, the absence of SRF seemed again to be a predictive factor of a worse visual outcome (OR, 0.848; 95\% CI, 0.759-0.948; $p=0.004)$, whereas intact EZ continuity was a predictive factor of a better final BCVA (OR, 1.137; 95\% CI, 1.015-1.273; $p=0.027$ ). 
TABLE 2: CDVA (log MAR) and CRT $(\mu \mathrm{m})$ as mean \pm standard deviation (range) at baseline and after 12 months with regard to naïve status and indication for surgery.

\begin{tabular}{|c|c|c|c|c|c|c|}
\hline \multirow[b]{2}{*}{ Parameter } & \multicolumn{2}{|c|}{ All eyes } & \multicolumn{2}{|c|}{ ERM } & \multicolumn{2}{|r|}{ RRD } \\
\hline & $\begin{array}{l}\text { Naïve } \\
(n=18)\end{array}$ & $\begin{array}{l}\text { Pretreated } \\
(n=43)\end{array}$ & $\begin{array}{l}\text { Naïve } \\
(n=15)\end{array}$ & $\begin{array}{l}\text { Pretreated } \\
(n=31)\end{array}$ & $\begin{array}{l}\text { Naïve } \\
(n=3)\end{array}$ & $\begin{array}{l}\text { Pretreated } \\
(n=12)\end{array}$ \\
\hline $\begin{array}{l}\text { CDVA at } \\
\text { baseline }\end{array}$ & $\begin{array}{c}0.82 \pm 0.32 \\
(0.2-1.3)\end{array}$ & $\begin{array}{c}0.55 \pm 0.27 \\
(0.1-1.1)\end{array}$ & $\begin{array}{c}0.87 \pm 0.30 \\
(0.4-1.3)\end{array}$ & $\begin{array}{c}0.55 \pm 0.28 \\
(0.1-1.2)\end{array}$ & $\begin{array}{c}0.57 \pm 0.4 \\
(0.2-1.0)\end{array}$ & $0.54 \pm 0.25(0.2-1.0)$ \\
\hline $\begin{array}{l}\text { CDVA at } \\
12 \mathrm{M}\end{array}$ & $\begin{array}{c}0.59 \pm 0.33 \\
(0.1-1.2)\end{array}$ & $\begin{array}{c}0.43 \pm 0.28 \\
(0-1.2)\end{array}$ & $\begin{array}{c}0.57 \pm 0.33 \\
(0.1-1.1)\end{array}$ & $\begin{array}{c}0.41 \pm 0.25 \\
(0.1-1.0)\end{array}$ & $\begin{array}{c}0.70 \pm 0.36 \\
(0.3-1.0)\end{array}$ & $0.49 \pm 0.35(0-1.2)$ \\
\hline$p$ value & $0.008^{*}$ & $0.003^{*}$ & $<0.0001^{\dagger}$ & $0.004^{*}$ & $0.655^{*}$ & $0.410^{\dagger}$ \\
\hline $\begin{array}{l}\text { CRT at } \\
\text { baseline }\end{array}$ & $\begin{array}{c}472.4 \pm 129.4 \\
(285-677)\end{array}$ & $\begin{array}{c}507.2 \pm 114.9 \\
(346-645)\end{array}$ & $\begin{array}{c}485.9 \pm 133.3 \\
(285-677)\end{array}$ & $\begin{array}{c}525.1 \pm 121.0 \\
(350-645)\end{array}$ & $\begin{array}{c}405.0 \pm 98.8 \\
(302-499)\end{array}$ & $460.9 \pm 85.2(346-639)$ \\
\hline $\mathrm{CRT}$ at $12 \mathrm{M}$ & $\begin{array}{c}380.2 \pm 109.9 \\
(225-660)\end{array}$ & $\begin{array}{c}365.5 \pm 102.5 \\
(243-733)\end{array}$ & $\begin{array}{c}377.6 \pm 104.3 \\
(225-660)\end{array}$ & $\begin{array}{c}365.7 \pm 111.7 \\
(243-733)\end{array}$ & $\begin{array}{c}393.3 \pm 161.9 \\
(245-566)\end{array}$ & $365.1 \pm 78.1(250-510)$ \\
\hline$p$ value & $0.031^{*}$ & $<0.0001^{*}$ & $0.017^{\dagger}$ & $<0.0001^{*}$ & $1.0^{*}$ & $0.08^{\dagger}$ \\
\hline
\end{tabular}

CDVA: corrected distance visual acuity; CRT: central retinal thickness; ERM: epiretinal membrane; RRD: rhegmatogenous retinal detachment. *Wilcoxon test; ${ }^{\dagger}$ paired $t$-test.

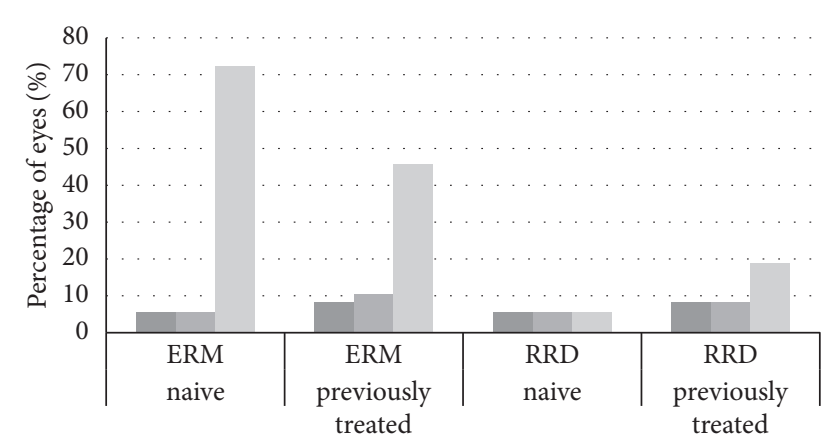

Indication for surgery and naive status

$$
\begin{aligned}
& \text { Loss }>1 \text { line } \\
& \text { No change } \\
& \text { Gain }>1 \text { line }
\end{aligned}
$$

FIgURe 3: Percentage of eyes that lost or gained $>1$ line of CDVA or remained stable with regard to naïve status and indication for surgery. Naïve ERM seems more likely to gain CDVA and less likely to lose CDVA or remain stable in comparison with previously treated eyes. On the other hand, RRD eyes seem more likely to gain CDVA, if previously treated. ERM: epiretinal membrane, RRD: rhegmatogenous retinal detachment, and CDVA: corrected distance visual acuity.

3.4. Adverse Events. No serious adverse events, such as vitreous hemorrhage, endophthalmitis, or dislocation of the implant in the anterior chamber, were associated with dexamethasone injection. An elevated intraocular pressure (IOP), defined as pressure over $25 \mathrm{mmHg}$ measured at any time after DEX injection, was assessed in $18 \%$ of the cases. These patients received topical IOP lowering treatment, but none required additional glaucoma surgery.

\section{Discussion}

The efficacy of DEX in the treatment of PCME has been shown in several studies after cataract surgery $[13,31,34]$, in two large cohorts in vitrectomized eyes after surgery for various underlying pathologies $[30,31]$, and, recently, in a small cohort after surgery for RRD with macular involvement [33]. However, most of these studies have focused on the development of CRT and BCVA after DEX injection and its adverse effects. The EPISODIC-2 investigation was the first study that evaluated predictive factors, such as preoperative existing risk factors, naïve status, and age, with regard to a better visual outcome after DEX treatment. Recently, one study has focused on OCT biomarkers for predicting the functional outcome at two and four months after one DEX injection for the treatment of diabetic macular edema [35].

The pharmacokinetics of the $0.7 \mathrm{mg}$ DEX implant is similar in vitrectomized and nonvitrectomized eyes $[15,18]$. DEX improves visual acuity, decreases central foveal thickness, reduces the functional and anatomical recurrence of PCME after cataract surgery in nonvitrectomized eyes $[13,31]$, and also shows positive results in the treatment of PCME in vitrectomized eyes [21, 26, 27, 29, 36, 37] without any increased risk of adverse effects [20].

The results of our study reveal the positive functional and morphological effects of DEX in vitrectomized eyes after surgery for ERM or RRD. BCVA improved in $76.1 \%$ of the ERM eyes and in $46.7 \%$ of RRD eyes. This was combined with a decrease of CRT at the last follow-up. The improvements in both BCVA and CRT confirm the positive effect of DEX in the treatment of PCME in vitrectomized eyes, as previously reported in several studies [29-31, 33, 35].

Interestingly, the mean number of DEX injections in our study reduced progressively over the follow-up period reflecting the efficacy of DEX, regardless of the indication for surgery. These results are in accordance with the study of Hattenbach et al. [30], in which 17 of 39 eyes required a second DEX injection and 8 of 17, a third treatment. However, in that study [30], only 6 eyes had a longer followup (>200 days), no improvement of CRT was noted, and only a slight improvement of BCVA was reported. Furthermore, three of the eyes were later treated with fluocinolone acetate implant. No other information was provided with regard to OCT morphology prior to the DEX injection in their investigation.

In our study, the mean number of required DEX injections did not seem to be affected by naïve status, and this status did not seem to increase the probability of a better 
TABLE 3: Baseline predictors of final visual outcome after 12 months after the first DEX injection.

\begin{tabular}{|c|c|c|c|c|c|}
\hline Baseline measure & $\begin{array}{c}\text { CDVA loss }>1 \text { line } n \\
(\%)\end{array}$ & $\begin{array}{c}\text { CDVA gain or loss } \leq 1 \text { line } \\
n(\%)\end{array}$ & $\begin{array}{c}\text { CDVA gain }>1 \text { line } n \\
(\%)\end{array}$ & $p$ value & OR $(95 \% \mathrm{CI})^{*}$ \\
\hline $\begin{array}{l}\text { Baseline CDVA } \\
(\log \mathrm{MAR} \pm \mathrm{SD})^{\dagger}\end{array}$ & $0.47 \pm 0.34$ & $0.50 \pm 0.26$ & $0.70 \pm 0.30$ & 0.001 & $1.485(1.171-1.884)$ \\
\hline $\begin{array}{l}\text { Indication for surgery } \\
\text { ERM } \\
\text { RRD }\end{array}$ & $\begin{array}{l}5 / 46(11) \\
4 / 15(27)\end{array}$ & $\begin{array}{l}6 / 46(13) \\
4 / 15(27) \\
\end{array}$ & $\begin{array}{l}35 / 46(76) \\
7 / 15(46) \\
\end{array}$ & 0.046 & $1.168(1.003-1.360)$ \\
\hline $\begin{array}{l}\text { Lens status } \\
\text { Phakic } \\
\text { Pseudophakic } \\
\end{array}$ & $\begin{array}{l}3 / 27(11) \\
6 / 34(18) \\
\end{array}$ & $\begin{array}{l}5 / 27(19) \\
5 / 34(15) \\
\end{array}$ & $\begin{array}{l}19 / 27(70) \\
23 / 34(67) \\
\end{array}$ & 0.820 & $1.015(0.890-1.159)$ \\
\hline $\begin{array}{l}\text { Naïve status } \\
\text { Nä̈ve } \\
\text { Previously treated } \\
\end{array}$ & $\begin{array}{l}2 / 18(11) \\
7 / 43(16) \\
\end{array}$ & $\begin{array}{l}2 / 18(11) \\
8 / 43(19) \\
\end{array}$ & $\begin{array}{l}14 / 18(78) \\
28 / 43(65) \\
\end{array}$ & 0.853 & $0.946(0.871-1.137)$ \\
\hline $\begin{array}{l}\text { IRF/cysts } \\
\text { Absence of IRF/cysts } \\
\text { Presence of IRF/cysts }\end{array}$ & $\begin{array}{l}1 / 8(12) \\
7 / 53(13)\end{array}$ & $\begin{array}{l}2 / 8(25) \\
8 / 53(15)\end{array}$ & $\begin{array}{c}5 / 8(63) \\
38 / 53(72)\end{array}$ & 0.465 & $1.055(0.915-1.216)$ \\
\hline $\begin{array}{l}\text { SRF } \\
\text { Absence of SRF } \\
\text { Presence of SRF } \\
\end{array}$ & $\begin{array}{l}8 / 43(19) \\
0 / 18(0)\end{array}$ & $\begin{array}{l}9 / 43(21) \\
1 / 18(6)\end{array}$ & $\begin{array}{l}26 / 43(60) \\
17 / 18(94)\end{array}$ & 0.043 & $0.860(0.743-0.995)$ \\
\hline $\begin{array}{l}\text { EZ continuity } \\
\text { Completely continuous } \\
\text { Partially disrupted } \\
\text { Completely disrupted } \\
\end{array}$ & $\begin{array}{l}4 / 12(33) \\
3 / 31(10) \\
1 / 18(6) \\
\end{array}$ & $\begin{array}{l}2 / 12(17) \\
3 / 31(10) \\
5 / 18(28) \\
\end{array}$ & $\begin{array}{l}6 / 12(50) \\
25 / 31(80) \\
12 / 18(66)\end{array}$ & 0.209 & $1.094(0.951-1.257)$ \\
\hline $\begin{array}{l}\text { Onset of PCME to surgery } \\
<3 \text { months } \\
>3 \text { months }\end{array}$ & $\begin{array}{l}7 / 38(18) \\
2 / 23(9) \\
\end{array}$ & $\begin{array}{c}3 / 38(8) \\
7 / 23(30)\end{array}$ & $\begin{array}{l}28 / 38(74) \\
14 / 23(61)\end{array}$ & 0.827 & $0.985(0.871-1.137)$ \\
\hline $\begin{array}{l}\text { Time between surgery and first } \\
\text { DEX } \\
<6 \text { months } \\
>6 \text { months }\end{array}$ & $\begin{array}{l}6 / 36(17) \\
3 / 25(12)\end{array}$ & $\begin{array}{l}7 / 36(19) \\
3 / 25(12)\end{array}$ & $\begin{array}{l}23 / 36(64) \\
19 / 25(76)\end{array}$ & 0.982 & $0.999(0.901-1.107)$ \\
\hline
\end{tabular}

CDVA: corrected distance visual acuity; CI: confidence interval; ERM: epiretinal membrane; RRD: rhegmatogenous retinal detachment; IRF: intraretinal fluid; SRF: subretinal fluid; EZ: ellipsoid zone; PCME: postoperative cystoid macular edema; DEX: dexamethasone intravitreal implant. ${ }^{*}$ Odds ratio (OR) for a patient presenting with a gain of CDVA $>1$ line at 12 months when baseline measure is increased by 1 value. ${ }^{\dagger}$ For every less line ( +0.1 log MAR) of baseline BCVA, a patient was more likely to gain $>1$ line in CDVA at 12 months after initial DEX injection.

final visual outcome. In contrast, in the EPISODIC-2, naïve eyes were reported only to need one DEX injection during the follow-up period [31]. However, EPISODIC-2 study involved 31 vitrectomized eyes (23 ERM and 8 RRD), and only 14 out of 100 enrolled eyes were naïve. Additionally, no information was provided regarding the surgery type of the naïve eyes. Nevertheless, with regard to the hypothesis of the EPISODIC-2 study, namely, that the kind of surgery responsible for PCME seems to be an important factor, the results of our study also indicate that surgery plays an important role in the final visual outcome after DEX treatment for PCME. However, we could find no influence of the preoperative lens status on the final visual outcome. Furthermore, the early ( $<3$ months) or late ( $>3$ months) onset of PCME after surgery and prompt (within 6 months after surgery) or delayed (after 6 months after surgery) treatment with DEX injection did not seem to have any predictive value with respect to the final functional outcome. In the ERM group, the mean BCVA improved the most in eyes that received one injection and progressively decreased with an increasing number of injections. However, in the RRD group, the mean BCVA showed greater improvement in eyes that received two injections. Interestingly, the mean
BCVA improved the most in naïve eyes after 2 injections and in pretreated eyes after three injections.

To date, the efficacy of DEX in vitrectomized eyes for the treatment of recalcitrant PCME after RRD repair has been investigated in one retrospective study that included 17 eyes [33]. However, major differences exist between that previous study and our cohort. Thanos et al. evaluated patients with recalcitrant PCME, and all eyes had RRD with macular involvement; most of them were complex cases including PVR (12/17) and silicone oil use (8/17), and some cases underwent vitrectomy combined with scleral buckling [33]. In our cohort, none of the included eyes had macular involvement, all underwent only one surgery during the follow-up period, no silicon oil was used, and no scleral buckling was performed. Therefore, in our cohort, the mean BCVA at the baseline was better, and the CRT was lower. Similarly, the small number of patients that developed an elevated IOP ( 2 eyes) in our study could be treated adequately with topical IOP-lowering medication. The average of 4 injections over the follow-up period in the study of Thanos et al. reflects the complexity of the enrolled cases in comparison with the 1.3 injections of our study over the 12month follow-up period. In our cohort, 12 eyes received only 
one DEX injection, 2 eyes received two injections, and 1 eye received three injections, indicating the high efficacy of DEX in majority of the cases.

Up to now, only one study has evaluated the predictive value of morphological structures of OCT after treatment with DEX for DME [35]. To our knowledge, our study is the first to evaluate the predictive value of a variety of factors, such as OCT biomarkers, indication for surgery, lens status, and baseline BCVA in the treatment of PCME with DEX in vitrectomized eyes. Subretinal fluid was present in 19 eyes at baseline and only in 5 eyes after 12 months, whereas EZ continuity was intact in 14 eyes at baseline and in 31 eyes one year later. Interestingly, the absence of SRF and a better baseline BCVA seemed to be predictive factors of a worse visual outcome.

The only significant adverse effect that has been reported after DEX injections is the elevation of IOP. In our study, $18 \%(11 / 61)$ of the patients showed a raise of IOP over $25 \mathrm{mmHg}$ and needed topical therapy. Most of the studies published to date record a similar prevalence of IOP elevation after DEX implantation, whereas Chin et al. have reported a higher percentage (26.9\%) [38]. We have observed no luxation of DEX in the anterior chamber, although all the patients of our cohort were pseudophakic (PC-IOL in the bag) and vitrectomized at the time of the injections.

In this study, we have investigated the efficacy of DEX in the treatment of PCME in vitrectomized eyes after ERM removal or RRD repair. Our results suggest that DEX should be considered as the first-line treatment option. The improvement of BCVA, the reduction of CRT, and the low incidence of IOP elevation strengthen the previously published data concerning the safety and efficacy of DEX in such cases [25]. Furthermore, the analysis of the morphological parameters of the OCT reveals the structural effect of DEX on the retinal layers and provides the predictive value of several variables on the final visual outcome. The decreasing need for further DEX injections over the follow-up time and the high mean BCVA improvement after the first DEX injection, especially in ERM cases, support the idea that DEX should be considered as the first-line therapy in the treatment of PCME. Furthermore, naïve status and lens status at vitrectomy do not seem to play a crucial role in the functional outcome.

The retrospective design of this study is its major limitation. Additionally, the nonequality of the two different groups (ERM and RRD) can be considered as a disadvantage. A further drawback is the fact that naïve eyes were only $29.5 \%$ of the cohort, whereas the remaining eyes had been previously treated and some of them were unresponsive. However, all previously treated eyes had experienced the same treatment, which included either parabulbous steroids, the same NSAID eye drops (nepafenac $1 \mathrm{mg} / \mathrm{ml} 3 \mathrm{x}$ daily), or a combination of them, offering a high homogeneity in the previously treated eyes. Moreover, we followed up all patients over a period of 12 months, evaluated BCVA, CRT, IOP elevation, lens status, and OCT biomarkers and recorded the odds ratios and predictive factors of the better visual outcome after treatment with DEX. We enrolled only uncomplicated patients who underwent one surgery during the follow-up time, thereby excluding other factors that might play a significant role in the development of PCME after vitrectomy.

In conclusion, this study demonstrates the efficacy and safety of DEX in the treatment of PCME in vitrectomized eyes after ERM removal or RRD repair and presents factors that have a predictive value for better functional outcome one year after the first DEX injection. The integrity of the EZ layer and the lower baseline BCVA seem to be predictive factors for better visual acuity, whereas the absence of SRF appears to be a predictive factor of worse final visual acuity. Naïve status and lens status at vitrectomy do not seem to play a significant role in that respect. Further studies with longer follow-up and a wider spectrum of diagnosis may reveal further predictive factors. Moreover, although an association between ERM, CME prior to vitrectomy, and the higher prevalence of PCME is known $[1,39]$, the ERM stage and OCT morphology at the time of vitrectomy should be investigated for their predictive value on the final visual outcome.

\section{Data Availability}

The data used to support the findings of this study are available from the corresponding author upon request.

\section{Conflicts of Interest}

The authors declare that they have no conflicts of interest.

\section{Authors' Contributions}

Efstathios Vounotrypidis and Sigrid Freissinger contributed equally on this work.

\section{Acknowledgments}

A. Wolf, K. U. Kortuem, and T. Herold report receiving personal fees from Allergan, but outside the scope of the submitted work

\section{References}

[1] C. J. Chu, R. L. Johnston, C. Buscombe, A. B. Sallam, Q. Mohamed, and Y. C. Yang, "Risk factors and incidence of macular edema after cataract surgery a database study of 81984 eyes," Ophthalmology, vol. 123, no. 2, pp. 316-323, 2016.

[2] S. Guo, S. Patel, B. Baumrind et al., "Management of pseudophakic cystoid macular edema," Survey of Ophthalmology, vol. 60, no. 2, pp. 123-137, 2015.

[3] A. B. Reese, I. S. Jones, and W. C. Cooper, "Macular changes secondary to vitreous traction," Transactions of the American Ophthalmological Society, vol. 64, no. 3, pp. 123-134, 1967.

[4] J. R. Wolter, "The histopathology of cystoid macular edema," Albrecht von Graefes Archiv fr Klinische und Experimentelle Ophthalmologie, vol. 216, no. 2, pp. 85-101, 1981.

[5] H. von Lany, S. Mahmood, C. R. H. James et al., "Displacement of nuclear fragments into the vitreous complicating phacoemulsification surgery in the UK: clinical features, outcomes and management," British Journal of Ophthalmology, vol. 92, no. 4, pp. 493-495, 2008. 
[6] H. N. Shelsta and L. M. Jampol, "Pharmacologic therapy of pseudophakic cystoid macular edema," Retina, vol. 31, no. 1, pp. 4-12, 2011.

[7] M. Packer, J. Lowe, and H. Fine, "Incidence of acute postoperative cystoid macular edema in clinical practice," Journal of Cataract \& Refractive Surgery, vol. 38, no. 12, pp. 21082111, 2012.

[8] L. H. P. Wielders, J. S. A. G. Schouten, B. Winkens et al., "European multicenter trial of the prevention of cystoid macular edema after cataract surgery in nondiabetics: ESCRS PREMED study report 1," Journal of Cataract \& Refractive Surgery, vol. 44, no. 4, pp. 429-439, 2018.

[9] B. Katzung, G. P. Chrousos, S. Masters, and A. Trevor, "Chapter. Adrenocorticosteroids \& adrenocortical antagonists," Basic \& Clinical Pharmacology \& Toxicology, vol. 39, pp. 592-607, 1995.

[10] M. Ritter, S. Sacu, G. Matt et al., "Use of systemic steroid after successful macular surgery in eyes with epiretinal membrane: a randomized, controlled clinical study," Eye, vol. 25, no. 10, pp. 1284-1293, 2011

[11] B. W. McCuen, M. Bessler, Y. Tano, D. Chandler, and R. Machemer, "The lack of toxicity of intravitreally administered triamcinolone acetonide," American Journal of Ophthalmology, vol. 91, no. 6, pp. 785-788, 1981.

[12] H.-S. Chin, T.-S. Park, Y.-S. Moon, and J.-H. Oh, "Difference in clearance of intravitreal triamcinolone acetonide between vitrectomized and nonvitrectomized eyes," Retina, vol. 25, no. 5, pp. 556-560, 2005.

[13] W. Mayer, S. Kurz, A. Wolf et al., "Dexamethasone implant as an effective treatment option for macular edema due to Irvine-Gass syndrome," Journal of Cataract \& Refractive Surgery, vol. 41, no. 9, pp. 1954-1961, 2016.

[14] T. Brynskov, C. S. Schmidt Laugesen, J. Halborg, H. Kemp, and T. L. Lykke Sorensen, "Longstanding refractory pseudophakic cystoid macular edema resolved using intravitreal $0.7 \mathrm{mg}$ dexamethasone implants," Clinical Ophthalmology, vol. 7, pp. 1171-1174, 2013.

[15] J.-E. Chang-Lin, J. A. Burke, Q. Peng et al., "Pharmacokinetics of a sustained-release dexamethasone intravitreal implant in vitrectomized and nonvitrectomized eyes," Investigative Opthalmology \& Visual Science, vol. 52, no. 7, pp. 4605-4609, 2011.

[16] B. D. Kuppermann, M. S. Blumenkranz, J. A. Haller et al., "Randomized controlled study of an intravitreous dexamethasone drug delivery system in patients with persistent macular edema," Archives of Ophthalmology, vol. 125, no. 3, pp. 309-317, 2007.

[17] J. A. Haller, F. Bandello, R. Belfort et al., "Randomized, shamcontrolled trial of dexamethasone intravitreal implant in patients with macular edema due to retinal vein occlusion," Ophthalmology, vol. 117, no. 6, pp. 1134-1146, 2010.

[18] J.-E. Chang-Lin, M. Attar, A. A. Acheampong et al., "Pharmacokinetics and pharmacodynamics of a sustained-release dexamethasone intravitreal implant," Investigative Opthalmology \& Visual Science, vol. 52, no. 1, p. 80, 2011.

[19] A. Degoumois, J. Akesbi, C. Laurens et al., "Efficacy of intravitreal dexamethasone implants in macular edema excluding venous occlusions: results for a cohort of 80 patients," Journal Français d'Ophtalmologie, vol. 38, no. 2, pp. 126-133, 2015.

[20] M. D. Medeiros, M. Alkabes, R. Navarro, J. Garcia-Arumí, C. Mateo, and B. Corcóstegui, "Dexamethasone intravitreal implant in vitrectomized versus nonvitrectomized eyes for treatment of patients with persistent diabetic macular edema,"
Journal of Ocular Pharmacology and Therapeutics: The Official Journal of the Association for Ocular Pharmacology and Therapeutics, vol. 30, no. 9, pp. 709-716, 2014.

[21] A. H. Shaikh, M. R. Petersen, R. A. Sisk, R. E. Foster, C. D. Riemann, and D. M. Miller, "Comparative effectiveness of the dexamethasone intravitreal implant in vitrectomized and non-vitrectomized eyes with macular edema secondary to central retinal vein occlusion," Ophthalmic Surgery, Lasers and Imaging Retina, vol. 44, no. 1, pp. 28-33, 2013.

[22] P. J. Banerjee, A. Quartilho, C. Bunce et al., "Slow-release dexamethasone in proliferative vitreoretinopathy: a prospective, randomized controlled clinical trial," Ophthalmology, vol. 124, no. 6, pp. 757-767, 2017.

[23] M. C. Gillies, L. L. Lim, A. Campain et al., "A randomized clinical trial of intravitreal bevacizumab versus intravitreal dexamethasone for diabetic macular edema: the BEVORDEX study," Ophthalmology, vol. 121, no. 12, pp. 2473-2481, 2014.

[24] P. Bansal, A. Agarwal, V. Gupta, R. Singh, and A. Gupta, "Spectral domain optical coherence tomography changes following intravitreal dexamethasone implant, Ozurdex ${ }^{\circledR}$ in patients with uveitic cystoid macular edema," Indian Journal of Ophthalmology, vol. 63, no. 5, pp. 416-422, 2015.

[25] M. Reibaldi, A. Longo, M. Fallico et al., "Widening use of dexamethasone implant for the treatment of macular edema," Drug Design, Development and Therapy, vol. 11, pp. 23592372, 2017.

[26] D. S. Boyer, D. Faber, S. Gupta et al., "Dexamethasone intravitreal implant for treatment of diabetic macular edema in vitrectomized patients," Retina, vol. 31, no. 5, pp. 915-923, 2011.

[27] A. Zheng, E. K. Chin, D. R. P. Almeida, S. H. Tsang, and V. B. Mahajan, "Combined vitrectomy and intravitreal dexamethasone (ozurdex) sustained-release implant," Retina, vol. 36, no. 11, pp. 2087-2092, 2016.

[28] L. S. Taney, C. R. Baumal, and J. S. Duker, "Sustained-release dexamethasone intravitreal implant for persistent macular edema after vitrectomy for epiretinal membrane," Ophthalmic Surgery, Lasers and Imaging Retina, vol. 46, no. 2, pp. 224228, 2015.

[29] C. Furino, F. Boscia, N. Recchimurzo, C. Sborgia, and G. Alessio, "Intravitreal dexamethasone implant for refractory macular edema secondary to vitrectomy for macular pucker," Retina, vol. 34, no. 8, pp. 1612-1616, 2014.

[30] L.-O. Hattenbach, C. Springer-Wanner, H. Hoerauf et al., "Intravitreal sustained-release steroid implants for the treatment of macular edema following surgical removal of epiretinal membranes," Ophthalmologica, vol. 237, no. 4, pp. 232-237, 2017.

[31] D. Bellocq, V. Pierre-Kahn, F. Matonti et al., "Effectiveness and safety of dexamethasone implants for post-surgical macular oedema including irvine-gass syndrome: the EPISODIC-2 study," British Journal of Ophthalmology, vol. 101, no. 3, pp. 333-341, 2017.

[32] G. Guidi, G. Casini, G. Ripandelli et al., "Residual intraretinal edema after 25-gauge vitrectomy and macular pucker removal: is intraoperative sustained-release dexamethasone a real treatment option?" Retina, vol. 38, no. 5, pp. 993-999, 2018.

[33] A. Thanos, B. Todorich, Y. Yonekawa et al., "Dexamethasone intravitreal implant for the treatment of recalcitrant macular edema after rhegmatogenous retinal detachment repair," Retina, vol. 38, no. 6, pp. 1084-1090, 2018.

[34] D. Bellocq, J. Korobelnik, C. Burillon et al., "Effectiveness and safety of dexamethasone implants for post-surgical macular 
oedema including Irvine-Gass syndrome: the EPISODIC study," British Journal of Ophthalmology, vol. 99, no. 7, pp. 979-983, 2015.

[35] D. Zur, M. Iglicki, C. Busch et al., "OCT biomarkers as functional outcome predictors in diabetic macular edema treated with dexamethasone implant," Ophthalmology, vol. 125, no. 2, pp. 267-275, 2018.

[36] A. Klamann, K. Böttcher, P. Ackermann, G. Geerling, M. Schargus, and R. Guthoff, "Intravitreal dexamethasone implant for the treatment of postoperative macular edema," Ophthalmologica, vol. 236, no. 4, pp. 181-185, 2016.

[37] N. Merkoudis and E. Granstam, "Treatment of postoperative cystoid macular oedema with dexamethasone intravitreal implant in a vitrectomized eye-a case report," Acta Ophthalmologica, vol. 91, no. 3, pp. 238-239, 2013.

[38] E. K. Chin, D. R. P. Almeida, G. Velez et al., "Ocular hypertension after intravitreal dexamethasone (ozurdex) sustained-release implant," Retina, vol. 37, no. 7, pp. 1345-1351, 2017.

[39] R. Frisina, S. J. Pinackatt, M. Sartore et al., "Cystoid macular edema after pars plana vitrectomy for idiopathic epiretinal membrane," Graefe's Archive for Clinical and Experimental Ophthalmology, vol. 253, no. 1, pp. 47-56, 2014. 